\title{
Liver Disorders in Inflammatory Bowel Disease
}

Authors:

Macarena Klein, ${ }^{1,2}$ *Paulina Núñez, ${ }^{2,3}$ Constanza Bay, ${ }^{4}$ Carolina Pizarro, ${ }^{5}$ Rocío Sedano, ${ }^{6}$ Rodrigo Quera ${ }^{6}$

1. Department of Gastroenterology, Faculty of Medicine, University of Chile, Santiago, Chile

2. San Juan de Dios Hospital, Santiago, Chile

3. Inflammatory Bowel Disease Unit, Department of Gastroenterology, Faculty of Medicine, University of Chile, Santiago, Chile

4. Department of Pediatrics, Faculty of Medicine, Pontifical Catholic University of Chile, Santiago, Chile

5. Hepatology Unit, Department of Gastroenterology, Faculty of Medicine, University of Chile, Santiago, Chile

6. Inflammatory Bowel Disease Program, Department of Gastroenterology, Clínica Las Condes, Santiago, Chile

*Correspondence to paulinanunez@gmail.com

Disclosure: $\quad$ The authors have declared no conflicts of interest.

Received: $\quad 19.02 .2020$

Accepted: $\quad 18.03 .2020$

Keywords: $\quad$ Extraintestinal manifestations, hepatotoxicity, inflammatory bowel disease (IBD), primary sclerosing cholangitis (PSC).

Citation:

EMJ Hepatol. 2020;8[1]:26-32.

\section{Abstract}

Abnormal liver tests are frequent in patients with inflammatory bowel disease. These may occur at the time of diagnosis or throughout the course of the disease. There are multiple aetiologies, such as concomitant diseases and extraintestinal manifestations of the same disease, primary sclerosing cholangitis being the most characteristic. Other aetiologies include adverse reactions to the drugs used in the treatment of these patients. This review will evaluate the different causes of liver test abnormalities.

\section{INTRODUCTION}

Hepatobiliary diseases constitute some of the most common extraintestinal problems of inflammatory bowel diseases (IBD) reported in ulcerative colitis (UC) and Crohn's disease (CD). Approximately $50 \%$ of patients with IBD will present a transient elevation of liver tests during long-term follow up. ${ }^{1}$ Non-alcoholic fatty liver disease (NAFLD) is the most common cause of impaired liver tests. Primary sclerosing cholangitis (PSC) is a liver disease more specifically associated with IBD, mainly

UC. ${ }^{2,3}$ Other related diseases are autoimmune hepatitis, primary biliary cholangitis ( $P B C)$, choledocholithiasis, hepatic amyloidosis, portal vein thrombosis, and drug-induced liver injury (DILI), among others. ${ }^{4}$ Clinicians must carry out a complete evaluation to determine the aetiology of abnormal liver tests, the possible association with a disease related to IBD, and their clinical relevance. The main objective of this review was to describe the main hepatobiliary manifestations related to IBD. 


\section{Liver Function Test Alteration}

The transient or persistent elevation of liver tests is frequent in IBD. In a recent study of 306 patients with IBD, $19.6 \%$ presented with abnormal liver test results. ${ }^{5}$ In up to $60.0 \%$ of the patients the alterations were mild and spontaneously returned to normal values. The most frequent cause of transient alteration in liver tests is secondary to DILI (34.1\%), while fatty liver is the most frequent cause of persistent transaminase alteration and even chronic liver disease $(65.4 \%){ }^{1}$

\section{PRIMARY SCLEROSING CHOLANGITIS}

PSC is characterised by chronic inflammation that affects the intra and/or extrahepatic bile ducts. PSC can cause segmental stenosis, saccular dilatations, different degrees of fibrosis, and in advanced stages, even liver cirrhosis. ${ }^{6}$

\section{Epidemiology}

The incidence of PSC is estimated at 0.9 and 0.5 per 100,000 inhabitants per year for North America and Europe, respectively. ${ }^{7}$ It usually occurs in men, with a median age of diagnosis of 41 years. ${ }^{8}$ Approximately $50-80 \%$ of patients with PSC have concomitant IBD, most often UC. Nevertheless, only $5 \%$ of patients with IBD develop PSC. Incidence varies if the diagnosis is radiologic or histopathologic. In a study that included 255 patients with IBD that underwent abdominal surgery and liver biopsy, $12.8 \%$ presented findings compatible with PSC. Of these, only $24.1 \%$ had alterations of liver function tests. ${ }^{9}$ In another study of 756 patients with IBD, 24 patients (7.5\%) had PSC-compatible lesions in the magnetic resonance cholangiography (MRCP). Only seven (2.2\%) of these had a previous PSC diagnosis by clinical findings and/or impaired liver tests. These data suggest that the prevalence may be underestimated, since up to two-thirds of the patients may be asymptomatic. ${ }^{10}$

\section{Clinical Findings, Diagnosis, and Complications}

Patients can be asymptomatic or present multiple symptoms that can be intermittent, such as fatigue, pruritus, fever, night sweats, and pain in the upper right quadrant. The laboratory finding of a cholestatic pattern (elevation of alkaline phosphatase and $\mathrm{V}$-glutamyl transferase) is characteristic. The study of choice is MRCP, given it is a non-invasive exam with high sensitivity and specificity. ${ }^{11,12}$ Typical findings of PSC include multifocal segmental stenosis with saccular dilations, which produce a classic appearance known as 'string beads'. The European Crohn's and Colitis Organisation (ECCO) consensus recommends restricting the use of endoscopic retrograde cholangiopancreatography only in cases of need for intervention as an indication for dilatation due to imaging and/or diagnostic cytological findings. ${ }^{13}$

Liver biopsy is reserved in cases of diagnostic doubt or if small duct PSC is suspected. Characteristic findings are obliterating fibrosis of small bile ducts with concentric periductal fibrosis in an 'onion skin' pattern. Causes of secondary sclerosing cholangitis must be excluded, such as infection, immunodeficiency, ischaemia, pancreatic disease, or diseases related to IgG4. The presence of PSC associated with IBD correlates with a specific IBD phenotype. ${ }^{14,15}$ Loftus et al. ${ }^{16}$ described a series of 71 patients with IBD. Evidence of backwash ileitis was found in $51 \%$ of the PSCIBD patients compared with $7 \%$ in the control group (IBD only). Rectum preservation was found in $52 \%$ and $6 \%$ of patients, respectively. ${ }^{16}$ PSC associated with UC mainly presents as extensive colitis beyond the splenic flexion in $90 \%$ of cases. There is greater involvement of the right colon, with rectum preservation, frequent refluxileitis, and increased risk of colorectal carcinoma (CRC), which persists after liver transplantation. ${ }^{5,16}$ When PSC associates with CD, the course of the IBD tends to be more benign. The predominant phenotype is the inflammatory type of colon, which may or may not have terminal ileum involvement. Stenosing and fistulising phenotypes are less frequent. ${ }^{14,17}$ The combination of PSC with IBD is associated with complications such as cholangitis, cholecystolithiasis, osteoporosis, fat-soluble vitamin deficiency, and steatorrhoea. ${ }^{18,19}$ These patients also have an increased risk of developing malignancies, mainly cholangiocarcinoma (CCA) and CRC. ${ }^{10}$ 


\section{Primary Sclerosing Cholangitis and Colon Cancer}

Patients with IBD have a significantly increased risk of developing $\mathrm{CRC}$, mainly because of the pro-neoplastic effect secondary to chronic intestinal inflammation. The risk is greater if there is an association with PSC. ${ }^{20}$ In 77 patients with PSC-IBD, CRC was detected in $7.8 \%$ versus $2.3 \%(p=0.016)$ in the control group (UC without PSC). ${ }^{13}$ Interestingly, in PSC-UC, all colorectal tumours were located proximal to the splenic flexion. In UC without PSC, tumour location was predominantly in the left colon (100\% versus $40 \%$ ).

Additional risk factors for the development of CRC include the duration of the disease and the extent of IBD (pancolitis). In a comparative study, 46 of 273 patients with PSC (223 with UC and 50 with $C D$ ), it was established that patients with UC had a $56 \%$ higher risk of developing CRC compared to CD. ${ }^{21}$

\section{Primary Sclerosing Cholangitis and Cholangiocarcinoma}

The possibility of developing CCA in patients with PSC is $10 \%$. The risk is $400-1,400$ times higher than that of the general population, ${ }^{12,22}$ and is even higher if PSC associates with IBD. ${ }^{23}$ CCA is mainly associated with intra and extrahepatic PSC, with some cases reported in small duct PSC..$^{24,25}$ Over half of patients with PSC and CCA are diagnosed at an advanced stage, in part because of the challenges of achieving an early diagnosis. Therefore, the diagnosis of CCA in patients with PSC requires a high index of suspicion and active surveillance. ${ }^{12}$

\section{Treatment}

No therapy has been shown to prevent liver transplantation, CCA, or death. ${ }^{8}$ The use of ursodeoxycholic acid (15-20 mg/ $\mathrm{kg} /$ day) is associated with an improvement in the cholestatic pattern. It has not been demonstrated to prevent the progression of the disease: the reason why the American Academy for the Study of Liver Disease (AASLD) does not recommend it. ${ }^{24}$
Liver transplantation is the only therapy that can cure PSC. Patients with PSC and end-stage liver disease or disabling symptoms (intractable pruritus or repeated cholangitis) should be considered for liver transplantation. ${ }^{33,17}$

\section{Follow-Up}

Due to the higher risk of colorectal cancer, ECCO guidelines ${ }^{12}$ recommend surveillance colonoscopy in patients with PSC and IBD at diagnosis and every 1 to 2 years after that. Chromoendoscopy with targeted biopsies is the surveillance strategy of choice. In patients with PSC without evidence of IBD, colonoscopy is recommended every 5 years. Screening PSC patients for CCA is a rational approach due to their increased risk of this neoplasia. Ultrasound imaging assessment of the biliary tree (sensitivity: 57\%; specificity: $94 \%$ ) or MRI/ MRCP (sensitivity: 89\%; specificity: 75\%) in combination with CA 19-9 every 6-12 months, seems to be the right approach. Experts recommend MRI for CCA surveillance, as it has higher sensitivity than ultrasound. ERCP should not be considered for surveillance. ${ }^{26,27}$

\section{OTHER HEPATIC MANIFESTATIONS IN PATIENTS WITH INTESTINAL BOWEL DISEASE}

\section{Non-Alcoholic Fatty Liver Disease}

NAFLD is a chronic liver disorder, characterised by the presence of steatosis in $>5 \%$ of hepatocytes. Current data suggest an increase in the prevalence of NAFLD in patients with IBD and is now one of the most frequent hepatic manifestations in IBD. The prevalence of NAFLD is $6.7-35.5 \%$ in patients with UC and 7.8-9.5\% in $C D .{ }^{28}$ The range of incidence depends on the diagnostic method used. A controlled study analysed 928 IBD patients, 7.2\% had NAFLD diagnosed with abdominal imaging. BMI and prevalence of metabolic syndrome were greater in NAFLD than patients without NAFLD. Risk factors for NAFLD in IBD included small bowel surgery (odds ratio [OR]: 3.7; 95\% confidence interval $[\mathrm{Cl}]$ : 1.5-9.3; $\mathrm{p}=0.005)$, hypertension (OR: 3.5; 95\% Cl: 1.5-8.1; $p=0.004$ ), obesity (OR: 2.1; 95\% Cl: 1.05-4.00; $p=0.035)$, and steroid use (OR:3.7; 95\% Cl: 1.5-9.3; $p=0.005) .{ }^{29}$ 
In addition to metabolic syndrome, the pathogenesis of NAFLD in the population with IBD may be more complex and involve specific risk factors for the disease, such as chronic inflammation, drug-induced hepatotoxicity, steroid exposure, malnutrition, and intestinal dysbiosis. ${ }^{30}$ Nonspecific guidelines for the evaluation of NAFLD in IBD have been established. Ultrasound is commonly used for the screening and evaluation in patients suspected of NAFLD. Non-invasive serum biomarker scores, such as the Fibrosis-4 calculator and NAFLD fibrosis score, have been validated for the assessment of fibrosis. There is also more information about using transient elastography (TE), which may assess the presence of advanced fibrosis. ${ }^{31}$ A specific treatment for the IBD population has not been evaluated. The current approach to NAFLD therapy is lifestyle and diet modification with the objective of a weight reduction of at least $7 \%$, which has been associated with a biochemical and histological improvement in patients with NAFLD. Pharmacological therapy should be evaluated case-by-case.

\section{Drug-Induced Liver Injury}

Drugs used in the treatment of IBD have accounted for some cases of DILI. These may be transient elevations of liver enzymes up to sporadic cases of clinically significant liver injury. ${ }^{32,33}$ Aminosalicylates report an estimate incidence of 3.2 cases per million prescriptions of DILI, including mild transaminase elevations, cholestatic pattern, and hypersensitivity reactions. ${ }^{34}$ The hepatoxic effects of thiopurines, azathioprine and 6-mercaptopurine, are primarily mediated by the metabolite 6-methylmercaptopurine. ${ }^{35}$ In adult patients with IBD starting thiopurines, the American Gastroenterological Association (AGA) have suggested routine thiopurine methyltransferase testing and monitoring of thiopurine metabolite to guide thiopurine dosing if IBD is active. ${ }^{35}$ Different patterns of hepatocellular, cholestatic, and mixed liver injury have been identified, characteristically being a more acute DILI. Longterm evolution can even lead to liver cirrhosis with portal hypertension secondary to vascular compromise (sinusoidal dilation, sinusoidal obstruction syndrome), and regenerative nodular hyperplasia. ${ }^{36}$ Methotrexate may cause acute liver test disturbances and prolonged use at cumulative doses greater than $1.5 \mathrm{~g}$ may develop macrovesicular steatosis and progressive fibrosis towards cirrhosis. Risk factors for fibrosis with methotrexate use include alcohol consumption, obesity, diabetes, and previous liver disease. ${ }^{37}$

Anti-TNF have been associated with four types of liver test alterations: 1) infusion hepatitis, which appears after two to five infusions, for which the alteration is usually transient and generally asymptomatic; 2) cholestatic, which can occur later; 3) de novo autoimmune hepatitis, with a hepatocellular pattern and the presence of antinuclear antibodies and other autoantibodies; and 4) reactivation of chronic hepatitis $B$, being necessary to test it before starting biological therapy. ${ }^{5,38}$ Vedolizumab is a gut-specific anti-integrin that binds a4- $\beta 7$ to MAdCAM1. In a systematic review it was shown that liver test alterations were not significant compared to placebo. ${ }^{39}$ Small molecules are an effective treatment in moderate-to-severe, immune-refractory or anti-TNF-failing UC. No significant alterations in liver tests have been reported regarding these. ${ }^{39}$ Table 1 shows the different hepatic damage patterns associated with drugs used in the treatment of IBD patients.

\section{Primary Biliary Cholangitis}

PBC is a chronic cholestatic nonsuppurative destructive cholangitis. Most patients are asymptomatic or have nonspecific symptoms such as fatigue or pruritus and it is not usually associated with IBD. Liberal et al. ${ }^{40}$ described a series of six patients with PBC-IBD where the majority were women without differences between UC or CD. ${ }^{40}$

\section{Autoimmune Hepatitis}

Autoimmune hepatitis (AlH) is a chronic liver disease characterised by alteration of transaminases, hypergammaglobulinaemia, and periportal hepatitis in liver biopsy. The prevalence in patients with IBD is low. It has been reported mainly in children with UC reaching up to $0.77 \%$. In patients with PSC, the prevalence is higher, where the overlap of $\mathrm{AlH}$ was observed in $10 \%$ of cases. ${ }^{41,42}$ The response to the treatment of $\mathrm{AlH}$ is not affected by the presence of IBD. 
Table 1: Hepatic damage patterns associated with drugs for intestinal bowel disease.

\begin{tabular}{|l|l|}
\hline Patterns & Associated Drug \\
\hline Hepatocellular & $\begin{array}{l}\text { Sulfasalazine, mesalamine, thiopurines, methotrexate, } \\
\text { anti-TNF }\end{array}$ \\
\hline Cholestatic & Sulfasalazine, mesalamine, anti-TNF \\
\hline Granulomatous hepatitis & Sulfasalazine, thiopurines \\
\hline $\begin{array}{l}\text { Vascular lesions (sinusoidal dilatation, peliosis hepatis, } \\
\text { veno-occlusive disease) }\end{array}$ & Thiopurines \\
\hline Nodular regenerative hyperplasia & Thiopurines \\
\hline Hepatic steatosis & Methotrexate, corticosteroids \\
\hline De novo autoimmune hepatitis & Anti-TNF \\
\hline Reactivation of chronic hepatitis B & Anti-TNF \\
\hline
\end{tabular}

\section{Pyogenic Liver Abscess}

Patients with IBD have a higher risk of pyogenic liver abscess than the general population. In a cohort study, the incidence was higher in IBD patients (6.72 IBD versus 4.06 per 10,000 person-year in non-IBD). ${ }^{43}$ The abscesses are often multiple in number and are located more frequently in the right hepatic lobe. Clinically they present with abdominal pain, jaundice, fever, diarrhoea, and in some cases, hepatomegaly. They are mainly associated with $C D$ due to transmural inflammation and may be secondary to direct extension of intra-abdominal abscess, pylephlebitis, or secondary to fistulising disease. Additional risk factors are diabetes and bile duct manipulation. ${ }^{44}$ Treatment does not differ from management in other clinical contexts. A guided antibiotic therapy should be administered according to cultures or drainage results, size, and evolution. ${ }^{30}$

\section{Hepatic Amyloidosis}

Secondary hepatic amyloidosis is a rare complication that has been reported in $0.90 \%$ of patients with CD. It is mainly described in cases of severe $C D$ with infectious complications and intestinal resection, and in $0.07 \%$ of patients with UC. ${ }^{45}$ Chronic inflammatory activity in the intestine contributes to the deposition of amyloid in the vessels and sinusoids of almost any organ, including the liver, which leads to asymptomatic hepatomegaly. The treatment is to decrease IBD activity.

\section{Granulomatous Hepatitis}

Granulomatous hepatitis is a rare complication of IBD, with a prevalence of less than $1 \%$, which is more frequent if associated with $C D$. It can be induced by drugs used in IBD treatment, such as mesalamine and sulfasalazine, and also with other concomitant autoimmune pathologies such as PBC and $\mathrm{AlH}$. The presence of non-calcified granulomas characterises it, occasionally with multinucleated cells, which are located both in the portal space and the lobules. Patients are usually asymptomatic, so suspicion should arise in the presence of a cholestatic pattern. ${ }^{46}$

\section{Portal Vein Thrombosis}

Patients with IBD have a known risk of increased thromboembolic disease, and the portal vein is a common place of thrombosis. Major risks have been described in the postoperative period of IBD and during exacerbations, although it may occur in patients in remission. In a Spanish retrospective study, $40 \%$ of patients who presented thrombotic episodes also had a proven prothrombotic genetic factor, the most frequent being hyperhomocysteinaemia. ${ }^{47}$ Therefore, the ECCO guidelines recommend an appropriate evaluation for both the underlying acquired prothrombotic conditions (related to IBD) and for hereditary thrombophilia. ${ }^{12}$ Treatment with anticoagulants is recommended according to general guidelines. 
to progressive severe diseases with poor prognosis. Therefore, in patients with IBD, liver tests should be routinely monitored, and a full Elevation of liver enzymes is frequent in diagnostic workup performed if they are altered patients with IBD. Causes are varied and (Figure 1). Differential diagnosis should always alterations range from slight increases include DILI.

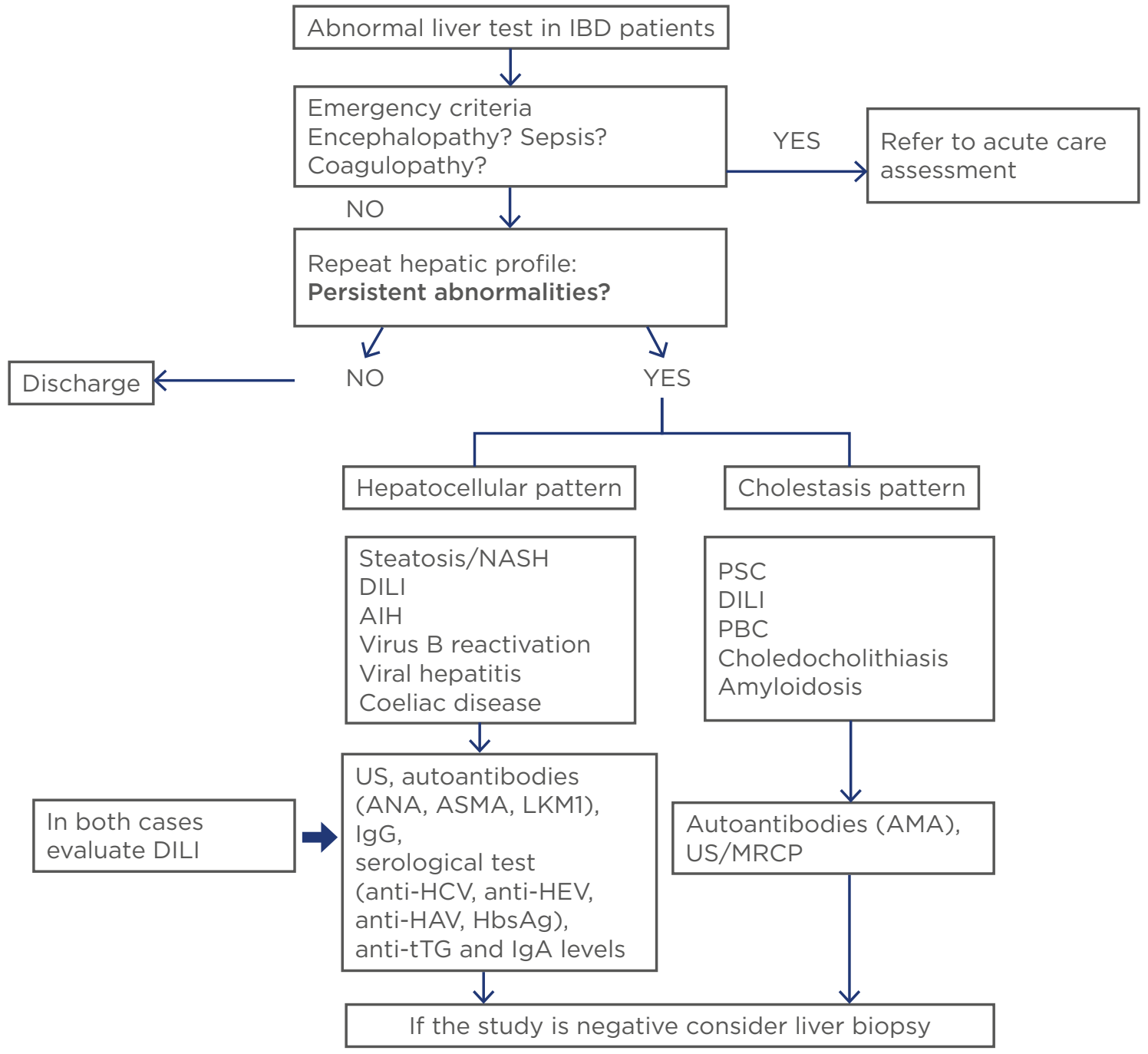

Figure 1: What should we do with abnormal liver test in inflammatory bowel disease? A stepwise approach for patients with abnormal liver test in IBD.

AlH: autoimmune hepatitis; ANA: antinuclear antibody; ASMA: anti-smooth muscle antibody; LKM1: liver kidney microsome antibody; AMA: antimitochondrial antibody; PBC: primary biliary cholangitis; DILI: drug-induced liver injury; HAV: hepatitis A virus; HCV: hepatitis C virus; HEV: hepatitis E virus; IBD: inflammatory bowel disease; MRCP: magnetic resonance cholangiography; NASH: non-alcoholic steatohepatitis; PSC: primary sclerosing cholangitis; tTG: tissue transglutaminase; US: ultrasound.

\section{References}

1. Loftus EV Jr et al. Interactions between chronic liver disease and inflammatory bowel disease. Inflamm Bowel Dis. 1997;3(4):288-302.
2. Cappello $M$ et al. Liver function test abnormalities in patients with inflammatory bowel diseases: a hospital-based survey. Clin Med
Insights Gastroenterol. 2014;7:25-31.

3. Fousekis FS et al. Hepatobiliary manifestations and complications in 
inflammatory bowel disease: a review. Gastroenterology Res. 2018;11(2):8394.

4. Yaccob A, Mari A. Practical clinical approach to the evaluation of hepatobiliary disorders in inflammatory bowel disease. Frontline Gastroenterol. 2019;10(3):309-15.

5. Silva $\mathrm{J}$ et al. Frequency of hepatobiliary manifestations and concomitant liver disease in inflammatory bowel disease patients. Biomed Res Int. 2019;2019:7604939.

6. Dyson JK et al. Primary sclerosing cholangitis. Lancet. 2018;391(10139):2547-59.

7. Weersma RK, Lindor KD. Shifting paradigms: what is the true prevalence and clinical course of primary sclerosing cholangitis? Gastroenterology. 2016;151(4):590-3.

8. Molodecky NA et al. Incidence of primary sclerosing cholangitis: a systematic review and meta-analysis. Hepatology. 2011;53(5):1590-9.

9. Miard $\mathrm{C}$ et al. Usefulness of systematic liver biopsy during a surgery for inflammatory bowel disease for the diagnosis of primary sclerosing cholangitis. Gastroenterology. 2018;154:S-20.

10. Lunder A et al. Prevalence of sclerosing cholangitis detected by magnetic resonance cholangiography in patients with long-term inflammatory bowel disease. Gastroenterology. 2016;151(4):660-9.

11. Nuñez FP et al. Primary sclerosing cholangitis and inflammatory bowel disease: intestine-liver interrelation. Gastroenterol Hepatol. 2019;42(5):316-25.

12. Aabakken $L$ et al. Role of endoscopy in primary sclerosing cholangitis: European Society of Gastrointestinal Endoscopy (ESGE) and European Association for the Study of the Liver (EASL) Clinical Guideline. Journal of Hepatology. 2017;66:1265-80

13. Harbord $M$ et al. The first European evidence-based consensus on extra-intestinal manifestations in inflammatory bowel disease. J Crohns Colitis. 2016;10(3):239-54.

14. Cordes F et al. Distinct disease phenotype of ulcerative colitis in patients with coincident primary sclerosing cholangitis: evidence from a large retrospective study with matched cohorts. Dis Colon Rectum. 2019;62(12):1494-504

15. Ricciuto A et al. The IBD and PSC Phenotypes of PSC-IBD. Curr Gastroenterol Rep. 2018;20(4):16.

16. Loftus EV Jr et al. PSC-IBD: a unique form of inflammatory bowel disease associated with primary sclerosing cholangitis. Gut. 2005;54(1):91-6.

17. Bleday $\mathrm{R}$ et al. Increased risk of early colorectal neoplasms after hepatic transplant in patients with inflammatory bowel disease. Dis Colon Rectum. 1993;36:908-12.
18. Chapman $\mathrm{MH}$ et al. British Society of Gastroenterology and UK-PSC guidelines for the diagnosis and management of primary sclerosing cholangitis. Gut. 2019;68(8):1356-78

19. Malik A et al. Preventive care in cholestatic liver disease: pearls for the specialist and subspecialist. Liver Res. 2019;3(2):118-27.

20. Palmela $\mathrm{C}$ et al. Inflammatory bowel disease and primary sclerosing cholangitis: a review of the phenotype and associated specific features. Gut Liver. 2018;12(1):17-29.

21. Navaneethan $U$ et al. Comparison of outcomes for patients with primary sclerosing cholangitis associated with ulcerative colitis and Crohn's disease. Gastroenterol Report (Oxf). 2016;4(1):43-9

22. Burak $\mathrm{K}$ et al. Incidence and risk factors for cholangiocarcinoma in primary sclerosing cholangitis. Am J Gastroenterol. 2004;99(3):523-6.

23. Razumilava N, Gores GJ. [Surveillance of cholangiocarcinoma in patients with primary sclerosing cholangitis: is it effective and justified?] Clin Liver Dis. 2016; 8(Suppl 1):S20-4. [Article in Spanish].

24. Chapman $\mathrm{R}$ et al. Diagnosis and management of primary sclerosing cholangitis. AASLD Practice Guideline. Hepatol. 2010;51(2):660-78.

25. Guerra I et al. Clinical characteristics, associated malignancies and management of primary sclerosing cholangitis in inflammatory bowel disease patients: a multicentre retrospective cohort study. J Crohns Colitis. 2019;13(12):1492-500.

26. Saffioti F et al. Pharmacological interventions for primary sclerosing cholangitis: an attempted network meta-analysis. Cochrane Database Syst Rev. 2017;3:CD011343.

27. Bowlus $\mathrm{C}$ et al. AGA clinical practice update on surveillance for hepatobiliary cancers in patients with primary sclerosing cholangitis: expert review. Clin Gastroenterol Hepatol. 2019;17(12):2416-22

28. Bargiggia $\mathrm{S}$ et al. Sonographic prevalence of liver steatosis and biliary tract stones in patients with inflammatory bowel disease: study of 511 subjects at a single center. J Clin Gastroenterol. 2003:36(5):417-20.

29. Sourianarayanane A et al. Risk factors of non-alcoholic fatty liver disease in patients with inflammatory bowel disease. J Crohns Colitis. 2013;7(8):e279-85.

30. Chao $\mathrm{C}$ et al. Co-existence of nonalcoholic fatty liver disease and inflammatory bowel disease: A review article. World J Gastroenterol. 2016;22(34):7727-34.

31. Palumvo $\mathrm{C}$ et al. Screening for nonalcoholic fatty liver disease in inflammatory bowel diseases: a cohort study using transient elastography. Inflamm Bowel Dis.
2019;25(1):124-33.

32. Shamberg L, Vaziri H. Hepatotoxicity of inflammatory bowel disease medications. J Clin Gastroenterol. 2018;52(8):674-84.

33. Rogler G. Gastrointestinal and live adverse effects of drugs used for treating IBD. Best Pract Res Clin Gastroenterol. 2010;24(2):157-65.

34. Stoschus B et al. Cholestasis associated with mesalazine therapy in a patient with Crohn's disease. J Hepatol. 1997;26(2):425-8.

35. Gisbert JP et al. Thiopurineinduced liver injury in patients with inflammatory bowel disease: a systematic review. Am J Gastroenterol. 2007;102:1518-27.

36. Feuerstein $\mathrm{J}$ et al. American Gastroenterological Association Institute Guideline on therapeutic drug monitoring in inflammatory bowel disease. Gastroenterology. 2017;153(3):827-34.

37. Tran-Minh M et al. Hepatic complications induced by immunosuppressants and biologics in inflammatory bowel disease. World J Hepatol. 2017;9(13):613-26.

38. French JB et al. Hepatotoxicity associated with the use of anti TNF a Agents. Drug Saf. 2016;39:199-208.

39. Lopetuso LR et al. Harmful effects and potential benefits of anti- tumor necrosis factor (TNF)-a on the liver. Int J Sci. 2018;19(8):2199.

40. Bye WA et al. Systematic review: the safety of vedolizumab for the treatment of inflammatory bowel disease. Aliment Pharmacol Ther 2017:46(1):3-15.

41. Agrawal M et al. JAK inhibitors safety in ulcerative colitis: practical implications. J Crohns Colitis. 2020; doi.org/10.1093/ecco-jcc/jjaaO17.

42. Kaplan GG et al. The burden of large and small duct primary sclerosing cholangitis in adults and children: a population-based analysis. Am J Gastroenterol. 2007;102(5):1042-9.

43. Lin JN et al. Pyogenic liver abscess in patients with inflammatory bowel disease: a nationwide cohort study. Liver Int. 2016;36(1):136-44.

44. Greenstein AJ et al. Amyloidosis and inflammatory bowel disease. A 50-year experience with 25 patients. Medicine (Baltimore). 1992;71(5):26170

45. McCluggage WG, Sloan JM. Hepatic granulomas in Northern Ireland: a thirteen-year review. Histopathology. 1994;25(3):219-28.

46. Landman $\mathrm{C}$ et al. Portomesenteric vein thrombosis in patients with inflammatory bowel disease. Inflamm Bowel Dis. 2013;19(3):582-9.

47. Liberal $\mathrm{R}$ et al. Primary biliary cholangitis in patients with inflammatory bowel disease. Clin Res Hepatol Gastroenterol. 2020;44(1):e59. 